\title{
CLIFFORD ALGEBRAS AND FAMILIES OF ABELIAN VARIETIES
}

\author{
I. SATAKE
}

To the memory of TADAsi Nakayama

In the arithmetic theory of automorphic functions on a symmetric bounded domain $\mathscr{D}=G / K$, as developed recently by Shimura and Kuga [2], [2a], it is important to consider a family of (polarized) abelian varieties on $\mathscr{D}$ obtained from a symplectic representation $\rho$ (defined over $\mathbf{Q}$ ) of $G$ (viewed as an algebraic group defined over $\mathbf{Q}$ ) satisfying a certain analyticity condition. Recently, I have determined completely such representations, reducing the problem to the case where $G$ is a $\mathbf{Q}$-simple group and where $\rho$ is a $\mathbf{Q}$-primary representation ([3], [4]). It has turned out that, besides the four standard solutions investigated already by Shimura, there exist two more non-standard solutions, one of which comes from a spin representation of the orthogonal group and thus gives a family of abelian varieties on a domain of type (IV). The purpose of this short note is to explain how one can construct most simply, starting from the "regular representation" of the corresponding Clifford algebra, examples of such families, including also the non-analytic case.

1. Let $V$ be an $n$-dimensional vector-space over $\mathbf{R}$, provided with a nondegenerate symmetric bilinear form $S$ of signature $(p, q)$. We denote by $C=$ $C(V, S)$ the corresponding Clifford algebra, by $C^{+}$(resp. $C^{-}$) its even (resp. odd) part, and define the "spin group" (or "reduced Clifford group" in the terminology of [1]) as follows:

$$
G=\left\{g \in C^{+} \mid g^{\ell} g=1, g V g^{-1}=V\right\},
$$

c denoting the canonical involution of $C$. We assume that $p, q>0, n=p+q>2$. The following Proposition is well-known (see e.g. [1], 2.9).

Proposition 1. 1) G is a connected semi-simple Lie group and the mapping

Received July 6, 1965. 
$\varphi$ defined by

$$
\varphi(g) x=g x g^{-1} \quad \text { for } g \in G, x \in V
$$

is a covering homomorphism of order 2 from $G$ onto the connected component of the identity of $S O(V, S)$ (the special orthogonal group).

2) Every maximal compact subgroup $K$ of $G$ can be written uniquely in the form

$$
K=G\left(V_{+}\right) G\left(V_{-}\right),
$$

where $V=V_{+}+V_{-}$is an orthognnal decomposition of $V$ such that $S \mid V_{+} \gg 0$, $S \mid V_{-} \ll 0$ and where $G\left(V_{+}\right), G\left(V_{-}\right)$denote the corresponding spin groups imbedded in a nxtural way in $G$.

Now, let $V=V_{+}+V_{-}$be an orthogonal decomposition of $V$ as described in Proposition 1 , and take an orthogonal basis $\left(e_{1}, \ldots, e_{n}\right)$ of $V$ with $S\left(e_{i}, e_{i}\right)$ $= \pm 1$ in such a way that $\left(e_{1}, \ldots, e_{p}\right)\left(\operatorname{resp} .\left(e_{p+1}, \ldots, e_{n}\right)\right)$ forms a basis of $V_{+}$ (resp. $V_{-}$); then, in $C$ one has

$$
\begin{aligned}
& e_{i}^{2}= \begin{cases}1 & \text { for } 1 \leq i \leq p, \\
-1 & \text { for } p+1 \leq i \leq n,\end{cases} \\
& e_{i} e_{j}=-e_{j} e_{i} \quad \text { for } i \neq j \text {. }
\end{aligned}
$$

Put further

$$
\begin{aligned}
& e_{+}=e_{1} \cdots e_{p}, \\
& e_{-}=e_{p+1} \cdots e_{n} ;
\end{aligned}
$$

$e_{+}$and $e_{-}$are elements in $C^{ \pm}$, which are uniquely determined up to a sign \pm 1 only by $V_{+}$and $V_{-}$.

PRoposition 2. The correspondence $x \rightarrow e_{+}^{-1} x^{\imath} e_{+}\left(=e_{-}^{-1} x^{e} e_{-}\right)$is a "positive" involution of $C^{+}$, i.e. the bilinear form $\operatorname{tr}\left(e_{+}^{-1} x^{2} e_{+} y\right)\left(x, y \in C^{+}\right)$is symmetric and positive-definite.

Proof. From $e_{+}^{\ell}= \pm e_{+}$, it is clear that this correspondence is an involution of $C^{+}$, so that the bilinear form $\operatorname{tr}\left(e_{+}^{-1} x^{t} e+y\right)$ is symmetric. To prove the positivity, let us put

$$
\begin{aligned}
& C_{+}^{+}=C^{+}\left(V_{+}\right) C^{+}\left(V_{-}\right), \\
& C_{-}^{+}=C^{-}\left(V_{+}\right) C^{-}\left(V_{-}\right),
\end{aligned}
$$

$C^{+}\left(V_{ \pm}\right)$(resp. $C^{-}\left(V_{ \pm}\right)$) denoting the even (resp. odd) part of the Clifford 
algebras $C\left(V_{ \pm}\right)$, imbedded in a natural way in $C=C(V)$. Then, one has $C^{+}=$ $C_{+}^{+}+C_{-}^{+}$(direct sum), and $e_{+}$and $e_{-}$commute elementwise with $C_{+}^{+}$and anticommute elementwise with $C_{-}^{+}$(i.e. one has $e_{ \pm} x=-x e_{ \pm}$for all $x \in C_{-}^{+}$). It follows that one has $\left(C_{+}^{+}\right)^{2}=\left(C_{-}^{+}\right)^{2}=C_{+}^{+}, C_{+}^{+} \cdot C_{-}^{+}=C_{-}^{+} \cdot C_{+}^{+}=C_{-}^{+}$and also that, writing $x, y \in C^{+}$in the form

$$
x=x_{+}+x_{-}, y=y_{+}+y_{-} \quad \text { with } x_{+}, y_{+} \in C_{+}^{+}, x_{-}, y_{-} \in C_{-}^{+},
$$

one has $e_{+}^{-1} x^{\ell} e_{+}=x_{+}^{\ell}-x_{-}^{\ell}$. Therefore one has

$$
\operatorname{tr}\left(e_{+}^{-1} x^{\imath} e_{+} y\right)=\operatorname{tr}\left(x_{+}^{\ell} y_{+}-x_{-}^{\imath} y_{-}\right) .
$$

From this it is easy to see that $\left\{e_{i_{1}} \cdots e_{i_{r}}\left(i_{1}<\cdots<i_{r}\right)\right\}$ forms an orthogonal basis of $C^{+}$with respect to this metric, and that one has $\operatorname{tr}\left(e_{+}^{-1} x^{e} e_{+} x\right)=1$ for all $x=e_{i_{1}} \cdots e_{i_{r}}$. This proves our assertion.

Remark. By a similar argument, one can show that the correspondence $x \rightarrow e_{+}^{-1} x^{\epsilon} e_{+}$(resp. $e_{-}^{-1} x^{\epsilon} e_{-}$) is a positive involution of $C$, if $p$ is odd (resp. if $q$ is even).

CoRollary. If $K$ is a maximal compact subgroup of $G$ corresponding to the orthogonal decomposition $V=V_{+}+V_{-}$and if $e_{ \pm}$is as defined by (4), then one has

$$
K=\left\{g \in G \mid e_{+} g=g e_{+}\left(\text {or } e_{-} g=g e_{-}\right)\right\} .
$$

Proof. Call $K^{*}$ the group defined by the right-hand side of (6). Then, from the above proof, one has $K^{*}=G \cap C_{+}^{+}$and so, by Proposition $1, K^{*}$ contains $K$. On the other hand, since $x \rightarrow e_{+}^{-1} x^{2} e_{+}$is a positive involution, $K^{*}=$ $\left\{g \in G \mid g^{\ell} e_{+} g=e_{+}\right\}$is compact. Therefore, from the maximality of $K$, one has $K=K^{*}$, q.e.d.

2. The notation being as in 1 , let us now consider the representation $\rho$ of $G$ in $C^{+}$defined by the left multiplication:

$$
\rho(g): x \longrightarrow g x \quad \text { for } x \in C^{+} .
$$

Fixing a maximal compact subgroup $K$ of $G$ once and for all, we consider the following problem.

Problem. Find all pairs $(A, I)$, where

1) $A$ is a non-degenerate alternating form on $C^{+}$which is invariant under all $\rho(g)(g \in G)$, and 
2) $I$ is a complex structure on $C^{+}$which is commutative with all $\rho(k)(k \in K)$, satisfying the following condition:

$$
\dot{A}(x, I y)\left(x, y \in C^{+}\right) \text {is symmetric and positive-definite. }
$$

If moreover one has a lattice $L$ in $C^{+}$such that

$$
A(x, y) \in \mathbf{Z} \quad \text { for all } x, y \in L,
$$

then one obtains an (analytic) family of polarized abelian varieties

$$
P_{g K}=\left(C^{+} / L, \rho(g) I \rho(g)^{-1}, A\right)
$$

parametrized by $g K \in G / K$, or rather by $\Gamma g K \in \Gamma \backslash G / K$, where $\Gamma=G_{L}=$ $\{g \in G \mid g L=L\}$ (cf. [2], [4]).

Proposition 3. All solutions of the above problem are given as follows:

$$
\begin{gathered}
A(x, y)=\operatorname{tr}\left(a x^{2} y\right), \\
I(x)=x b_{1}+e_{-} x b_{2},
\end{gathered}
$$

where $a$ is an invertible element in $C^{+}$such that $a^{b}=-a$ and $b_{1}$ and $b_{2}$ are elements in $C^{+}$and $C^{ \pm}$, respectively, satisfying the following conditions:

$$
\begin{gathered}
\left\{\begin{array}{l}
b_{1}^{2}+(-1)^{\frac{q(q+1)}{2}} b_{2}^{2}=-1, \\
b_{1} b_{2}+b_{2} b_{1}=0,
\end{array}\right. \\
\operatorname{tr}\left(b_{1} a x^{c} y\right)+\operatorname{tr}\left(b_{2} a x^{c} e_{-} y\right)\left(x, y \in C^{+}\right) \text {is symmetric and positive-definite. }
\end{gathered}
$$

Proof. Let $(A, I)$ be a solution of our problem. Then, first of all, there exists a uniquely determined (non-singular) linear transformation $f$ of $C^{+}$onto itself such that one has $A(x, y)=\operatorname{tr}\left(f(x)^{c} \cdot y\right)$. From the invariance of $A$ under the left multiplication by $G$, one obtains

$$
f(g x)=\left(g^{\ell}\right)^{-1} f(x)=g \cdot f(x) \quad \text { for all } g \in G, x \in C^{+} .
$$

Since the linear closure of $G$ is equal to $C^{+}$([1], II. 4.3, II. 5.1), this implies that one has $f(x y)=x f(y)$ for all $x, y \in C^{+}$. Hence, putting $f(1)^{c}=a$, one gets $f(x)=x a^{\imath}$, where $a$ is invertible and such that $a^{\imath}=-a$, because $A$ is nondegenerate and alternating. This proves (9). Next, since, by Proposition 1 , the linear closure of $K$ is $C_{+}^{+}=C^{+}\left(V_{+}\right) C^{+}\left(V_{-}\right)$, it follows from the commutativity of $I$ with the left multiplication by $K$ that one has

$$
I(x y)=x I(y) \quad \text { for all } x \in C_{+}^{+}, y \in C^{+},
$$


and so, in particular, $I(x)=x I(1)$ for $x \in C_{+}^{+}$. Let $u_{3}$ be any invertible element in $C_{-}^{+}$; then one has $I(x)=x u_{0}^{-1} I\left(u_{0}\right)$ for $x \in C_{-}^{+}$. Therefore, writing $x \in C^{+}$ in the form $x=x_{+}+x_{-}$with $x_{+} \in C_{+}^{+}, x_{-} \in C_{-}^{+}$, one has

$$
\begin{aligned}
I(x) & =I\left(x_{+}\right)+I\left(x_{-}\right) \\
& =x_{+} \cdot I(1)+x_{-} u_{0}^{-1} \cdot I\left(u_{0}\right) \\
& =\frac{1}{2}\left(x+e_{-} x e_{-}^{-1}\right) \cdot I(1)+\frac{1}{2}\left(x-e_{-} x e_{-}^{-1}\right) u_{0}^{-1} I\left(u_{0}\right) .
\end{aligned}
$$

Hence, putting $b_{1}=\frac{1}{2}\left(I(1)+\boldsymbol{u}_{0}^{-1} I\left(u_{0}\right)\right)\left(\in C^{+}\right), b_{2}=\frac{1}{2} e_{-}^{-1}\left(I(1)-u_{0}^{-1} I\left(u_{0}\right)\right)\left(\in C^{\mathrm{t}}\right.$ according as $q$ is even or odd), one gets (10). From $I^{2}=-1$ and $e_{-}^{2}=$ $(-1)^{\frac{q(q+1)}{2}}$ one gets (11), and from the condition (7) one gets (12), q.e.d.

ExAmpLe 1. In the case where $b_{1}=\lambda_{1} a^{-1}, b_{2}=\lambda_{2}\left(a e_{-}\right)^{-1}$ with non-zero real numbers $\lambda_{1}, \lambda_{2}$, the conditions (11), (12) reduce to the following:

$$
\left\{\begin{array}{l}
\boldsymbol{a} e_{-}=-e_{-} \boldsymbol{a}, \\
\boldsymbol{a}^{2}=\lambda_{2}^{2}-\lambda_{1}^{2}>0, \\
\lambda_{2}>0 .
\end{array}\right.
$$

ExAmple 2. In the case where $b_{1}=0, b_{2}=\lambda_{2}\left(a e_{-}\right)^{-1}$ with a non-zero real number $\lambda_{2}$, the conditions (11), (12) reduce to the following:

$$
\left\{\begin{array}{l}
a^{\iota} e_{-} a=\lambda_{2}^{2} e_{-}, \\
\lambda_{2}>0 .
\end{array}\right.
$$

ExAmple 3. In the case $p$ or $q=2$, the symmetric space $\mathscr{E}=G / K$ has a $G$-invariant complex structure and becomes an irreducible symmetric domain of type (IV). In this case, it is of particular significance to consider those solutions of our problem which further satisfy an "analyticity condition" $\left(H_{2}\right)$ saying that the induced map $\mathscr{D} \rightarrow \overline{\mathscr{J}}=\bar{G} / \bar{K}$ is complex analytic, where $\bar{G}$ $=S p\left(C^{+}, A\right)$ (the symplectic group) and $\bar{K}$ is a maximal compact subgroup of $\bar{G}$ defined by $\bar{K}=\left\{g \in \bar{G} \mid g I g^{-1}=I\right\}$. In the notation of [3], [4], this condition can also be expressed by saying that $d \rho\left(H_{0}\right)=\bar{H}_{0}$, where $H_{0}$ and $\bar{H}_{0}$ are respective $H$ elements for $G$ and $\bar{G}$. If one identifies the Lie algebra $g$ of $G$ with a certain linear subspace of $C^{+}$in a natural way, then one has $H_{0}= \pm \frac{1}{2} \boldsymbol{e}_{+}$ or $\pm \frac{1}{2} e_{-}$according as $p=2$ or $q=2$. On the other hand, one has $\bar{H}_{0}= \pm \frac{1}{2} I$. Thus, for instance, in the case $H_{0}=\frac{1}{2} e_{-}, \bar{H}_{0}=\frac{1}{2} I$, the condition $\left(H_{2}\right)$ says 
that $I(x)=e_{-} x$, i.e. $b_{1}=0, b_{2}=1$. The condition (12) then reduces to saying that $\operatorname{tr}\left(a x^{e} e-y\right)$ is symmetric and positive-definite, or in other words, that $a e$ - is a "positive" element with respect to the involution $x \rightarrow e_{-}^{-1} x^{e} e_{-}$, (which implies, besides the condition $a^{l}=-a$, that all the eigen-values of the linear transformation $x \rightarrow a e_{-} x\left(x \in C^{+}\right)$are positive real numbers).

Remark 1. If one puts

$$
\begin{aligned}
& \widetilde{G}=\left\{g \in C^{+} \mid g^{\ell} g=1\right\}, \\
& \widetilde{K}=\left\{g \in \widetilde{G} \mid g e_{-}=e_{-} g\right\},
\end{aligned}
$$

then the alternating form $A$ and the complex structure $I$ given in Proposition 3 are invariant also under the left multiplication by $\widetilde{G}$ and $\tilde{K}$, respectively. Therefore, one has actually a family of polarized abelian varieties $P_{g \tilde{K}}=$ $\left(C^{+} / L, g g^{-1}, A\right)$ parametrized by $g \widetilde{K} \in \tilde{\mathscr{D}}=\widetilde{G} / \widetilde{K}$, of which our family becomes a subfamily. It is not difficult to verify that the group $\widetilde{G}$ is of hermitian type, if and only if $p \equiv 2$ (4) or $q \equiv 2$ (4). In that case, identifying the Lie algebra $\widetilde{g}$ of $\widetilde{G}$ with $\left\{x \in C^{+} \mid x^{\ell}+x=0\right\}$, one sees that $\widetilde{g}=\widetilde{g} \cap C_{+}^{+}+\widetilde{g} \cap C_{-}^{+}$is a Cartan decomposition of $\widetilde{g}$ and an $H$-element for $\widetilde{G}$ is given by $\widetilde{H}_{0}= \pm \frac{1}{2} e_{+}$or $\pm \frac{1}{2} e_{-}$.

Remark 2. The simplest way to assure the measure-finiteness of the quotient space $T \backslash \mathscr{D}$ is to assume that $V$ and $S$ are both defined over $\mathbf{Q}$ and that $L$ is in $C_{\mathbf{Q}}^{+}=C^{+}\left(V_{Q}, S\right)$. The first condition implies that there exists a vector-space $V_{\mathbf{Q}}$ over $\mathbf{Q}$ contained in $V$ such that $V=V_{\mathbf{Q}} \otimes{ }_{\mathrm{Q}} \mathbf{R}$ and that $S$ is a canonical extension to $V$ of a bilinear form on $V_{\mathbf{Q}}$. In this case $G$ has a structure of an algebraic group defined over $\mathbf{Q}$ and $\Gamma=G_{L}$ is a so-called arithmetic subgroup of $G$. The condition (8) then implies that $A$ is also defined over $\mathbf{Q}$, or what amounts to the same by Proposition 3 , that $a \in C_{\mathbf{Q}}^{+}$.

Remark 3. If one denotes by $Z$ the center of $C^{+}$, one has

$$
Z \cong \begin{cases}\mathbf{R} & \text { if } n \equiv 1(2), \\ \mathbf{C} & \text { if } n \equiv 0, \frac{n}{2}+q \equiv 1 \quad(2), \\ \mathbf{R} \oplus \mathbf{R} & \text { if } n \equiv 0, \frac{n}{2}+q \equiv 0 \text { (2). }\end{cases}
$$

In the first and second cases, $C^{+}$is $\mathbf{R}$-simple and the representation $\rho$ is $\mathbf{R}$ primary (of type $\left(a_{1}\right)$ if $p-q \equiv \pm 1$ (8), of type $\left(a_{2}\right)$ if $p-q \equiv \pm 3(8)$, of type 
$\left(a_{3}\right)$ if $n \equiv 0$ (4) and $q \equiv 1$ (2), of type (b) if $n \equiv 2$ (4) and $\left.q \equiv 0(2)\right)$; in the third case, $C^{+}$is the direct sum of two central simple algebras $C^{+\prime}$ and $C^{+\prime \prime}$ over $\mathbf{R}$ and so $\rho$ has two $\mathbf{R}$-primary components (of type $\left(a_{1}\right)$ if $n \equiv 0$ (4) and $p-q \equiv 0$ (8), of type $\left(a_{2}\right)$ if $n \equiv 0$ (4) and $p-q \equiv 4$ (8), of type (s) if $n \equiv 2$ (4) and $q \equiv 1(2)$ ) (see [4]). In the third case, our solution $(A, I)$ decomposes in an obvious sense into the direct sum of two similar solutions $\left(A^{\prime}, I^{\prime}\right)$ and $\left(A^{\prime \prime}, I^{\prime \prime}\right)$ relative to $C^{+\prime}$ and $C^{+\prime \prime}$. In the case where $V$ and $S$ are defined over

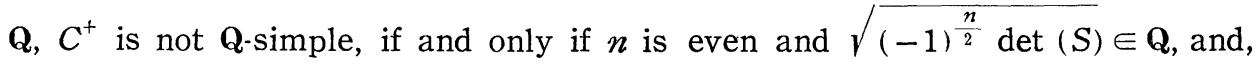
in that case, $\rho$ has two Q-primary components $\rho^{\prime}$ and $\rho^{\prime \prime}$. Then $L$, taken to be contained in $C_{\mathbf{Q}}^{+}$, is commensurable to a direct sum of two lattices $L^{\prime}$ and $L^{\prime \prime}$ in $C_{\mathbf{Q}}^{+\prime}$ and $C_{\mathbf{Q}}^{+\prime \prime}$, respectively. Thus our family of abelian varieties is isogeneous to the "Whitney sum" of two families $\left\{P_{g K}^{\prime}=\left(C^{+\prime} / L^{\prime}, \rho^{\prime}(g) I^{\prime} \rho^{\prime}(g)^{-1}, A^{\prime}\right)\right\}$ and $\left\{P_{g K}^{\prime \prime}=\left(C^{+\prime \prime} / L^{\prime \prime}, \rho^{\prime \prime}(g) I^{\prime \prime} \rho^{\prime \prime}(g)^{-1}, A^{\prime \prime}\right)\right\}(g \in G)$.

3. We denote by $\mathscr{P}\left(L, e_{-}, a, b_{1}, b_{2}\right)$ the family of polarized abelian varieties determined by the data $\left(L, e_{-}, a, b_{1}, b_{2}\right)$ as given in 2 . We shall now examine the condition under which two families of this form are equivalent. We recall that two families $\left\{\left(W / M, I_{z}, A\right)(z \in \mathscr{D})\right\},\left\{\left(W^{\prime} / M^{\prime}, I_{z}^{\prime}, A^{\prime}\right)\left(z^{\prime} \in \mathscr{D}^{\prime}\right)\right\}$ are called to be equivalent, if there exist a complex analytic isomorphism $\psi$ of $\mathscr{L}$ onto $\mathscr{D}^{\prime}$ and a linear isomorphism $\Psi$ of $W$ onto $W^{\prime}$ satisfying the following conditions :

$$
\begin{cases}\Psi(L)=L^{\prime}, & \\ \Psi \circ I_{z}=I_{\psi(z)}^{\prime} \circ \Psi & \text { for } z \in \mathscr{D}, \\ \mu A(x, y)=A^{\prime}(\Psi(x), \Psi(y)) & \text { for } x, y \in W,\end{cases}
$$

with a positive rational number $\mu$.

First of all, let $V=V_{+}^{\prime}+V_{-}^{\prime}$ be another orthogonal decomposition of $V$, and let $K^{\prime}$ and $e^{\prime}$ - be the corresponding maximal compact subgroup of $G$ and the element defined by (4). We assert that, if the system $\left(a, b_{1}, b_{2}\right)$ satisfies the conditions in Proposition 3 with respect to $e_{-}$, then so does the system also with respect to $\pm \boldsymbol{e}_{-}^{\prime}$ and the family $\mathscr{P}\left(L, \pm \boldsymbol{e}_{-}^{\prime}, \boldsymbol{a}, b_{1}, b_{2}\right)$ is equivalent to $\mathscr{P}\left(L, e_{-}, a, b_{1}, b_{2}\right)$. In fact, the only condition for $\left(a, b_{1}, b_{2}\right)$ which depends on the choice of $e_{-}$is (12). Since there exists $g_{1} \in G$ such that $K^{\prime}=g_{1}^{-1} K g_{1}$, $\pm \boldsymbol{e}_{-}^{\prime}=g_{1}^{-1} \boldsymbol{e}_{-} g_{1}$, the condition (12) relative to $\boldsymbol{e}_{-}$implies that

$$
\operatorname{tr}\left(b_{1} a x^{\ell} y\right)+\operatorname{tr}\left(b_{2} a x^{\ell}\left( \pm e_{-}^{\prime}\right) y\right)=\operatorname{tr}\left(b_{1} a\left(g_{1} x\right)^{\ell}\left(g_{1} y\right)\right)+\operatorname{tr}\left(b_{2} a\left(g_{1} x\right)^{\ell} e_{-}\left(g_{1} y\right)\right)
$$


is symmetric and positive-definite, i.e. (12) holds with respect to $\pm \boldsymbol{e}_{-}^{\prime}$, and vice-versa. Moreover, it is clear that, if one defines an analytic isomorphism $\psi$ of $G / K^{\prime}$ onto $G / K$ by $\psi: g K^{\prime} \rightarrow g g_{1} K$ and puts $\Psi=$ id., then the two families $\mathscr{P}\left(L, \pm \boldsymbol{e}_{-}^{\prime}, a, b_{1}, b_{2}\right)$ and $\mathscr{P}\left(L, e_{-}, a, b_{1}, b_{2}\right)$ are equivalent by $(\psi, \Psi)$, which proves our assertion. Therefore, in the following we shall fix $e_{-}$once and for all and write simply as $\mathscr{P}\left(L, a, b_{1}, b_{2}\right)$ instead of $\mathscr{P}\left(L, e_{-}, a, b_{1}, b_{2}\right)$.

Profosition $4^{11}$. Tuo families $\mathscr{P}\left(L, a, b_{1}, b_{2}\right)$ and $\mathscr{P}\left(L^{\prime}, a^{\prime}, b_{1}^{\prime}, b_{2}^{\prime}\right)$ are equivalent, if and only if there exist an element $g_{2}$ in $G$ and an invertible element $v$ in $C^{+}$such that one has

$$
\left\{\begin{array}{l}
L^{\prime}=g_{2} L v, \\
a^{\prime}=\mu v^{-1} a v^{-\imath}, \\
b_{i}^{\prime}=v^{-1} b_{i} v
\end{array}\right.
$$

with a positive rational number $\mu$.

Proof. Suppose that the two families are equivalent by $(\psi, \Psi)$. Then, first an analytic automorphism $\psi$ of $\mathscr{D}=G / K$ onto itself is given by a correspondence of the form

$$
\psi: g K \longrightarrow g_{2} g K
$$

with $g_{2} \in G$. Hence, putting $\Psi_{1}(x)=g_{2} x$ for $x \in C^{+}$, one sees that $\left(\psi, \Psi_{1}\right)$ defines an equivalence of $\mathscr{P}\left(L, a, b_{1}, b_{2}\right)$ to $\mathscr{P}\left(g_{2} L, a, b_{1}, b_{2}\right)$. Therefore, replacing $\Psi$ by $\Psi_{1}^{-1} \Psi$, one may assume from the beginning that $\psi=$ id.

Next, let us consider the set $\mathscr{L}$ of all linear transformations $\Phi$ of $C^{+}$ satisfying the condition

$$
\Phi\left(g \cdot I\left(g^{-1} x\right)\right)=g I^{\prime}\left(g^{-1} \Phi(x)\right)
$$

or what amounts to the same,

$$
\Phi\left(x b_{1}+g e_{-} g^{-1} x b_{2}\right)=\emptyset(x) \cdot b_{1}^{\prime}+g e_{-} g^{-1} \cdot \Phi(x) \cdot b_{2}^{\prime} .
$$

$\mathscr{L}$ is clearly a linear subspace of $\mathscr{L}\left(C^{+}\right)$, the vector-space of all linear endomorphisms of $C^{+}$into itself. Let us first consider the case where $n$ is odd. Then, $C^{+}$being central simple, $\mathscr{L}\left(C^{+}\right)$can be identified with $C^{+} \otimes C^{+}$by the

1) When the rationality condition as stated in Rem. 2 is satisfied, this Proposition is an easy consequence of a density theorem of Borel. 
linear isomorphism defined by

$$
\begin{gathered}
C^{+} \otimes C^{+} \ni u \otimes v \longrightarrow \Phi_{n, v} \in \mathscr{L}\left(C^{+}\right), \\
\Phi_{n, v}(x)=u x v \quad \text { for } x \in C^{+} .
\end{gathered}
$$

We make $g \in G$ operate on $C^{+} \otimes C^{+}$in the following manner :

$$
u \otimes v \longrightarrow\left(g u g^{-1}\right) \otimes v .
$$

Then, from the definition, $\mathscr{L}$ is clearly invariant under this operation of $G$. On the other hand, if one denotes by $C_{r}$ the homogeneous part of $C$ of degree $r$ (with respect to any orthogonal basis of $V$ ), then $C_{r} \otimes v$ (or: even, $v \in C^{+}, v \neq 0$ ) is a $G$-invariant subspace of $C^{+} \otimes C^{+}$, which is irreducible since the representation of $g \in G$ in this space is nothing else than the skew-symmetric tensor representation of degree $r$ of $\varphi(g) \in S O(V, S)$. Moreover, since $n$ is odd, two subspaces $C_{r} \otimes v, C_{r} \otimes v^{\prime}\left(r, r^{\prime}:\right.$ even $)$ are $G$-isomorphic if and only if $r=r^{\prime}$. Therefore $\mathscr{L}$ is a direct sum of subspaces of the form $C_{r} \otimes v$ with $v \in C^{+}$. Now if $C_{r} \otimes v_{1} \in \mathscr{L}$ with $v_{1} \neq 0$, then one has from (*)

$$
u x b_{1} v_{1}+u e_{-} x b_{2} v_{1}=u x v_{1} b_{1}^{\prime}+e_{-} u x v_{1} b^{\prime}
$$

for all $x \in C^{+}$. If $0<\boldsymbol{r} \leq \boldsymbol{n}$, then one can always find $u=\boldsymbol{e}_{i_{1}} \cdots e_{i_{r}}$ which is not commutative with $e_{-}$; then $\left\{\boldsymbol{u}, \boldsymbol{u} \boldsymbol{e}_{-}, \boldsymbol{e}_{-} \boldsymbol{u}\right\}$ are linearly independent, contradicting the above equality. Thus we should have $r=0$, and so $\mathscr{L} \subset 1 \otimes C^{+}$. Therefore one can write $\Psi$ in the form $\Psi(x)=x v$ with an invertible element $v$ in $C^{+}$. In the case where $\boldsymbol{n}$ is even, one has instead the linear isomorphism:

$$
\mathscr{L}\left(C^{+}\right) \cong C^{+} \otimes_{2} C^{+}+C^{-} \otimes_{2} C^{-}
$$

defined in the same way, $Z$ denoting the center of $C^{+}$; and the similar argument as above gives us again $\Psi(x)=x v$ with $v \in C^{+}$. Then, in either case, the conditions (14) with $g_{2}=1$ follow from (13) immediately. The converse ('if' part) is trivial, q.e.d.

It follows, in particular, that in the case where $q=2$ and $V$ and $S$ are defined over $\mathbf{Q}$ the number of equivalence-classes (with $\psi=\mathrm{id}$.) of the families of the form $\mathscr{P}(L, a, 1,0)$ for a fixed lattice $L$ in $C_{\mathbf{Q}}^{+}$is finite, as is easily seen from the reduction-theory of positive elements in an involutorial algebra.

Finally we remark that similar results can be obtained, whenever one has a group $G$ realized as a subgroup of the unitary group of an involutorial semisimple algebra $\mathfrak{A}$, such that the linear closure of $G$ coincides with $\mathfrak{A}$, and 
a representation $\rho$ of $G$ induced from the regular representation of $\mathfrak{A}$. Actually, Kuga [2] considered already the case where $G=S L(2, \mathbf{R})$ and where $\rho$ is a direct sum of a certain number of copies of the regular representation, with a beautiful application to the theory of automorphic forms with respect to the group of units of an indefinite quaternion algebra over $\mathbf{Q}$.

Addendum. The notation being as in the text, let $V$ and $S$ be both defined over $\mathbf{Q}$ and let $L$ be a lattice in $C_{\mathbf{Q}}$. In general, the (left) regular representation $\rho$ of $G$ in $C^{+}$being Q-reducible, the abelian variety $\mathscr{A}_{g K}=\left(C^{+} / L, \rho(g) I \rho(g)^{-1}\right)$ is not simple in the sense of isogeny. We shall add here some indications on the decomposition of $\mathscr{A}_{g K}$ in the most important special cases. Namely, we assume that the complex structure $I$ is given by $I(x)=e_{ \pm} x$ (which implies that $p$ or $q \equiv 2$ (mod. 4 )), and consider the following two extreme cases :

(i) the case where $g K$ is a 'generic' point of $\Gamma \backslash G / K$;

(ii) the case where $g K$ corresponds to a decomposition $V=V_{+}^{\prime}+V_{-}^{\prime}$ defined over $\mathbf{Q}$.

To begin with, it is clear that the endomorphism algebra $\operatorname{End}_{\mathrm{Q}}\left(\mathscr{A}_{g K}\right)=$ $\operatorname{End}\left(\mathscr{A}_{g K}\right) \otimes \mathbf{Q}$ of the abelian variety $\mathscr{A}_{g K}$ is the commutator algebra of $\rho(g) I \rho(g)^{-1}$ in the full linear endomorphism algebra $\mathscr{L}_{\mathrm{Q}}\left(C^{+}\right)$of $C^{+}$, where $C^{+}$is viewed merely as a vector-space defined over $\mathbf{Q}$. By definition, for a generic $g K, \operatorname{End}_{\mathrm{Q}}\left(\mathscr{A}_{g K}\right)$ coincides with the commutator algebra of $\left\{\rho(g) I \rho(g)^{-1} \mid g \in G\right\}$ in $\mathscr{L}_{\mathrm{Q}}\left(C^{+}\right)$, which by a similar argument as in the proof of Proposition 4 (or by using the density theorem of Borel) can be proved to be consisting of all right translations $x \rightarrow x v$ with $v \in C_{\mathbf{Q}}^{+}$. Thus one has

$$
\operatorname{End}_{\mathbf{Q}}\left(\mathscr{A}_{g K}\right) \cong C_{\mathbf{Q}}^{+} \text {. }
$$

From the well-known results on the structure of Clifford algebra (see Rem. 3), one can therefore conclude that

$$
\mathscr{A}_{g K} \sim \begin{cases}2^{\frac{n-1}{2}} \mathscr{A}_{1} \text { or } 2^{\frac{n-3}{2}} \mathscr{A}_{2} & \text { if } n \equiv 1(2), \\ 2^{\frac{n}{2}-1} \mathscr{A}_{3} & \text { if } n \equiv 0(2) \text { and } \sqrt{(-1)^{\frac{n}{2}} \operatorname{det}(S)} \notin \mathbf{Q}, \\ 2^{\frac{n}{2}-1} \mathscr{A}_{1}^{\prime} \times 2^{\frac{n}{2}-1} \mathscr{A}_{1}^{\prime \prime} \text { or } 2^{\frac{n}{2}-2} \mathscr{A}_{2}^{\prime} \times 2^{\frac{n}{2}-2} \mathscr{A}_{2}^{\prime \prime} \\ \text { if } n \equiv 0(2) \text { and } \sqrt{(-1)^{\frac{n}{2}} \operatorname{det}(S) \in \mathbf{Q},}\end{cases}
$$


where $\mathscr{A}_{1}$ (resp. $\left.\mathscr{A}_{1}^{\prime}, \mathscr{A}_{1}^{\prime \prime}\right)$ is a simple abelian variety of dimension $2^{\frac{n-3}{2}}$ (resp. $2^{\frac{n}{2}-2}$ ) without complex multiplication, $\mathscr{A}_{2}$ (resp. $\mathscr{A}_{2}^{\prime}, \mathscr{A}_{2}^{\prime \prime}$ ) is a simple abelian variety of dimension $2^{\frac{n-1}{2}}$ (resp. $2^{\frac{n}{2}-1}$ ) with endomorphism algebra isomorphic to a central quaternion algebra over $\mathbf{Q}$ (which depends only on $\mathbf{S}$ and not on $g K$ ), and $\mathscr{A}_{3}$ is a simple abelian variety of dimension $2^{\frac{n}{2}-1}$ with endomorphism algebra isomorphic to $\mathbf{Q}\left(\sqrt{(-1)^{\frac{n}{2}} \operatorname{det}(S)}\right)$. Here the notation $m \mathscr{A}$ stands for the direct product of $m$ copies of the abelian variety $\mathscr{A}$ and $\mathscr{A} \sim \mathscr{A}^{\prime}$ means that $\mathscr{A}$ and $\mathscr{A}^{\prime}$ are isogeneous to each other.

Let us now consider the case (ii). To simplify the notation, we shall assume $g=1$, and consider only the case where $I(x)=e_{+} x$. Since the decomposition $V=V_{+}+V_{-}$is defined over $\mathbf{Q}$, we can take an orthogonal basis $\left(\boldsymbol{e}_{1}^{\prime}\right.$, $\left.\ldots, e_{n}^{\prime}\right)$ of $V_{\mathrm{Q}}$ with $S\left(e_{i}^{\prime}, e_{i}^{\prime}\right)=\alpha_{i}$ in such a way that $\left(e_{1}^{\prime}, \ldots, e_{p}^{\prime}\right)$ (resp. $\left.\left(e_{p+1}^{\prime}, \ldots, e_{n}^{\prime}\right)\right)$ forms a basis of $\left(V_{+}\right)_{Q}\left(\operatorname{resp} .\left(V_{-}\right)_{Q}\right)$. Then, $e_{1}^{\prime} \cdots e_{p}^{\prime}$ being a scalar multiple of $e_{+}$, the commutator algebra of $I$ in $\mathscr{L}_{\mathrm{Q}}\left(C^{+}\right)$is equal to that of the left translation $x \rightarrow\left(e_{1}^{\prime} \cdots e_{p}^{\prime}\right) x$, which is defined over $\mathbf{Q}$. Hence, in view of $\left(e_{1}^{\prime} \cdots e_{p}^{\prime}\right)^{2}=(-1)^{\frac{p(p-1)}{2}} \alpha_{1} \cdots \alpha_{p}$, it follows that one has

$$
\operatorname{End}_{Q}\left(\mathscr{A}_{g K}\right) \cong M_{2^{n-2}}\left(\mathbf{Q}\left(\sqrt{(-1)^{\frac{p(p-1)}{2}} \alpha_{1} \cdots \alpha_{p}}\right)\right) .
$$

Therefore one concludes that

$$
\mathscr{A}_{g K} \sim 2^{n-2} E,
$$

where $E$ is an abelian variety of dimension 1 (i.e. an elliptic curve) with endomorphism algebra isomorphic to $\mathbf{Q}\left(\sqrt{(-1)^{\frac{p(p-1)}{2}} \alpha_{1} \cdots \alpha_{p}}\right)$.

In conclusion, we note that, applying a similar construction for the 'twisted' Clifford algebra obtained from a quaternionic skewhermitian form (see [4]), one may obtain a family of abelian varieties whose generic member is isogeneous to a direct product of simple abelian varieties with endomorphism algebra isomorphic to a central division algebra of dimension 16 .

\section{REFERENCES}

[1] Chevalley, C. C., The algebraic theory of spinors, Columbia Univ. Press, New York, 1954.

[2] Kuga, M., Fiber varieties over a symmetric space whose fibers are abelian varieties, I, II, Lecture Notes, Univ. of Chicago, 1963-64.

[2 a] Kuga, M. and Shimura, G., On the zeta function of a fibre variety whose fibres are 
abelian varieties, Ann. of Math., 82 (1965), 478-539.

[3] Satake, I., Holomorphic imbeddings of symmetric domains into a Siegel space, Amer, J. of Math., 87 (1965), 425-461.

[4] Satake, I., Symplectic representations of algebraic groups satisfying a certain analyticity condition, forthcoming.

University of Chicago 